\title{
Vor 20 Jahren: Rückblick auf den 13. Kongress der Gesellschaft für Bronchopneumonologie der DDR (zuletzt Gesellschaft für Pulmologie und Tuberkulose e. V.)
}

\author{
20 Years Ago: Looking Back at the 13th Congress of the Society for Bronchopneumology of the GDR \\ (later: Society for Pulmonology and Tuberculosis)
}

Autor

B. Wiesner

Bibliografie

DOI http://dx.doi.org/

10.1055/s-0030-1256253

Pneumologie 2011; 65:

189-190 @ Georg Thieme

Verlag KG Stuttgart · New York

ISSN 0934-8387

Korrespondenzadresse

Priv.-Doz. Dr.

Bernhard Wiesner

Robert-Koch-Allee 6

99438 Bad Berka

bernhard.wiesner@t-online.de
Der 52. Kongress der Deutschen Gesellschaft für Pneumologie und Beatmungsmedizin in Dresden gibt uns Anlass, an den letzten Kongress der Gesellschaft für Pulmologie und Tuberkulose e.V., die entsprechend dem Vereinsgesetz der Bundesrepublik aus der Gesellschaft für Bronchopneumonologie der DDR hervorgegangen war, zu erinnern. Auf diesem Kongress, der vom 20. bis 23 . März 1991 ebenfalls in Dresden stattfand, wurde Bilanz über die erfolgreiche Arbeit dieser Gesellschaft gezogen, hier wurde aber auch die Auflösung der Gesellschaft und die Rückführung in die Deutsche Gesellschaft für Pneumologie beschlossen.

Die Fachgesellschaft in der DDR wurde 1957 als „Wissenschaftliche Tuberkulosegesellschaft in der DDR“ gegründet mit dem Ziel, die Fortbildung der Ärzte auf dem Fachgebiet zu intensivieren, neue Forschungsergebnisse schnell umzusetzen, die Bevölkerung umfassend über die Krankheit aufzuklären und Einfluss zu nehmen auf die sozialen Erfordernisse bei der Betreuung der Kranken. Die Gründung der Gesellschaft in der DDR erfolgte nach längeren Verhandlungen im Konsens mit dem Vorstand der „Deutschen Tuberkulose-Gesellschaft“. Im Statut wurde festgeschrieben, dass die bisherigen Mitglieder und neu in diese Gesellschaft eintretenden Kollegen Mitglied in der „Deutschen Tuberkulose-Gesellschaft“ bleiben bzw. dort ebenfalls neu aufgenommen werden. Die alten Mitglieder der Gesellschaft fühlten sich ihr auch immer verbunden. Zum ersten Präsidenten wurde Adolf Tegtmeier (Bad Berka) gewählt. 1960 wurde er zum Vizepräsidenten der „Deutschen Tuberkulose-Gesellschaft“ gewählt, konnte das Amt des Präsidenten dann aber wegen der entstandenen politischen Situation nicht antreten.

Die Gesellschaft hat mit der Neuausrichtung des Fachgebietes auf unspezifische Lungenkrankheiten auch ihren Namen geändert. Mit den neuen Aufgaben wurden Arbeitsgruppen ins Leben gerufen, an deren Arbeit sich eine große Zahl der
Mitglieder aktiv beteiligte. In diesen Arbeitsgruppen wurden gemeinsam mit den leitenden Kliniken, insbesondere mit dem unter Leitung von Paul Steinbrück stehenden Forschungsinstitut für Lungenkrankheiten und Tuberkulose (FLT), Diagnostik- und Therapieempfehlungen erarbeitet, die damit von Anfang an auf eine breite Basis gestellt wurden, wodurch die Überführung in die medizinische Praxis wesentlich erleichtert werden konnte.

1967 hatte sich durch die schnellen Entwicklungen aus der Arbeitsgemeinschaft für Bronchologie die „Gesellschaft für Bronchologie“ gegründet, die alle an der Methodik interessierten Fachdisziplinen einschloss, sehr schnell für die Verbreitung der speziellen bronchologischen Techniken (Beatmungsbronchoskop, Bronchografie) als wichtiges diagnostisches und therapeutisches Instrument sorgte und über die Grenzen des Landes bekannt wurde. Sie war die erste medizinisch-wissenschaftliche Gesellschaft, die Mitglied einer internationalen Gesellschaft (Ass. Int. pour l'Etude des Bronches) wurde und 1970 deren Kongress in Dresden durchführte. 1976 erfolgte aber wieder die Zusammenführung dieser Gesellschaft mit der „Gesellschaft für Lungenkrankheiten und Tuberkulose in der DDR“ zur „Gesellschaft für Bronchopneumonologie der DDR“. Unter diesem Namen existierte sie bis 1990 .

Seit 1967 war die Gesellschaft eigenständiges Mitglied der IUAT, in deren Gremien eine Reihe von Ärzten und Wissenschaftlern mitarbeitete.

Höhepunkte der Arbeit waren die insgesamt 13 Kongresse, deren wissenschaftliche Leitung dem jeweiligen Präsidenten oblag. Diese Kongresse erfreuten sich stets eines großen Zuspruchs der Mitglieder und es war dank der internationalen Beziehungen immer möglich, auch ausländische Kollegen dazu einzuladen. Der 1. Kongress, der sich naturgemäß noch vorwiegend mit der Tuberkulose befasste, fand 1959 in Weimar statt. Hier kam aber auch schon mit Vorträgen zu speziellen 


\section{Tagung \\ der Gesellschaft für Pulmologie und Tuberkulose e.V.}

Dresden

21.-24. März 1991

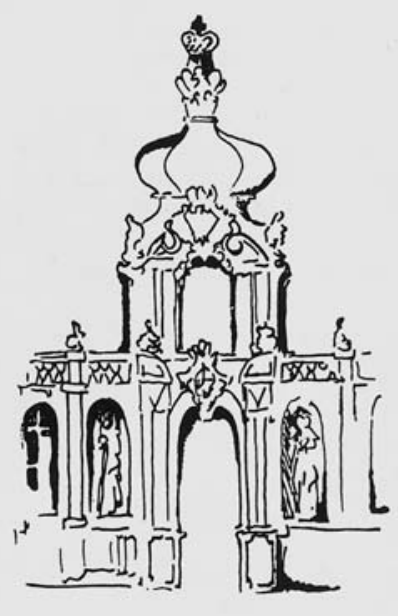

Abb. 1 Kronentor des Dresdener Zwingers. Deckblatt des Programms zum 13. Kongress der Gesellschaft für Bronchopneumonologie der DDR.

bronchologischen Themen zum Ausdruck, dass sich ein Wandel im Fachgebiet vollzog.

Die Themen der einzelnen Kongresse können hier nicht im Einzelnen besprochen werden, sie orientierten sich jeweils am aktuellen Stand der Forschungsergebnisse und an den praktischen Erfordernissen in der Organisation der Bekämpfung der Tuberkulose und der Lungenkrankheiten. Hervorgehoben werden soll aber der 10. Kongress. Er wurde 1982 anlässlich des 100. Jahrestages der Entdeckung des Tuberkuloseerregers durch Robert Koch gemeinsam mit der „Gesellschaft für Mikrobiologie und Epidemiologie der DDR" in Berlin durchgeführt, wo auch an historischem Ort, dem Hygiene-Institut der Kaiser-Wilhelm-Universität (Humboldt-Universität zu Berlin), in der Dorotheenstraße des epochalen Ereignisses gedacht wurde und eine Kranzniederlegung am Denkmal von Robert Koch auf dem Gelände der Charité erfolgte. Dieser Kongress führte erwartungsgemäß viele internationale Gäste nach Berlin, u.a. auch die damalige Generalsekretärin der IUAT, Annik Rouillon.

Das wissenschaftliche Programm des letzten Kongresses der Gesellschaft 1991 war auf folgende aktuelle Themen ausgerichtet:

- Epidemiologie und Prävention unspezifischer bronchopulmonaler Erkrankungen (W. Schilling, Berlin; Heidrun Behrendt, Düsseldorf)

- Epidemiologie und Prävention der Tuberkulose (Helga Scharkoff, Cottbus; R. Ferlinz, Mainz)

- Entzündliche Lungenkrankheiten/Pneumonien (B. Wiesner, Bad Berka)

- Chronische respiratorische Insuffizienz - ambulante Sauerstofftherapie (A. Wilke, Berlin; G. Liebetrau, Bad Berka)

- Asthma bronchiale (Jutta Adolph, Coswig; Gisela Kroemer, Pulsnitz).
Daneben wurden von den Arbeitsgruppen vier Symposien veranstaltet:

- Tumoren des Thorax (H. Dürschmied, Beelitz; C. Engelmann, Berlin)

- Respiratory mechanisms in the pulmonary vasculature and their significance for clinical treatment (A. Oddoy, Berlin)

- Interstitielle Lungenkrankheiten (H. Eckert, Berlin; E. Barthel, Waren/Müritz)

- Mucovizcidose (A. Ballin, Leipzig; Jutta Hein, Rostock)

Auch an diesem Kongress nahmen hochrangige Vertreter der IUAT, der deutschen, der österreichischen und der schweizerischen Fachgesellschaften teil. Mit großer Freude konnten wir viele Kollegen aus Russland, Polen, Ungarn und der Tschechoslowakei begrüßen, zu denen sich in den Jahren der deutschen Teilung sehr enge freundschaftliche Beziehungen entwickelt haben. Besonders betont werden muss aber, dass wir in Dresden auch unsere Freunde und Kollegen wieder trafen, die die DDR verlassen hatten und die jetzt problemlos an unserem Kongress teilnehmen konnten.

Für die Mitglieder war es nicht einfach, eine Entscheidung über das weitere Schicksal ihrer Gesellschaft zu treffen. Eine Auflösung der Gesellschaft wurde erst nach einer heftigen, durchaus auch von Resignation geprägten Grundstimmung geführten Diskussion, ob sie nicht als eigenständige Ostdeutsche Gesellschaft in den bestehenden Grenzen weiter existieren sollte, beschlossen, da eine einfache Fusion beider Gesellschaften nicht realisierbar war. Unsere Mitglieder fühlten sich durch ihre z. T. jahrzehntelange Mitarbeit in den Arbeitsgruppen, ihrer dort geleisteten wissenschaftlichen Arbeit und durch die im Laufe von Jahren entstandenen persönlichen Kontakte der Gesellschaft sehr eng verbunden. Den Mitgliedern wurde nach dem Beschluss zur Auflösung empfohlen, sich zur Mitgliedschaft in der Deutschen Gesellschaft für Pneumologie zu entscheiden, 57\% sind dieser Empfehlung gefolgt. Der Kongress wurde trotz der genannten Probleme mit einem Gesellschaftsabend im historischen Landhaus (früheres Ständehaus, Stadtmuseum Dresden), auf dem den Mitgliedern noch einmal eindringlich die Mitgliedschaft in der DGP empfohlen wurde, harmonisch beendet.

Nicht unerwähnt soll sein, dass der Gründungspräsident und der letzte Präsident der Gesellschaft leitende Mitarbeiter der Zentralklinik Bad Berka waren, die mit ihren hervorragenden Mitarbeitern und modernster technischer Ausrüstung einen wesentlichen Beitrag zur Bekämpfung der Tuberkulose, der unspezifischen Lungenkrankheiten und der Herzkrankheiten geleistet hat.

\section{Interessenkonflikt}

$\nabla$

Der Autor gibt an, dass kein Interessenkonflikt besteht. 